\title{
Five misconceptions in cancer diagnosis
}

\author{
William Hamilton
}

\begin{abstract}
Much investment has been put into facilities for early cancer diagnosis. It is difficult to know how successful this investment has been. New facilities for rapid investigation in the UK have not reduced mortality, and may cause delays in diagnosis of patients with lowrisk, or atypical, symptoms. In part, the failure of new facilities to translate into mortality benefits can be explained by five misconceptions. These are described, along with suggested research and organisational remedies. The first misconception is that cancer is diagnosed in hospitals. Consequently, secondary care data have been used to drive primary care decisions. Second, GPs are thought to be poor at cancer diagnosis, yet the type of education on offer to improve this may not be what is needed. Third, symptomatic cancer diagnosis has been downgraded in importance with the introduction of screening, yet screening identifies only a small minority of cancers. Fourth, pressure is put on GPs to make referrals for those with an individual high risk of cancer disenfranchising those with 'low-risk but not no-risk' symptoms. Finally, considerable nihilism exists about the value of early diagnosis, despite considerable observational evidence that earlier diagnosis of symptomatic cancer is beneficial.

Keywords

cancer; diagnosis; primary health care.
\end{abstract}

W Hamilton, MD, FRCP, FRCGP, consultant senior lecturer, National School for Primary Care Research, Academic Unit of Primary Health Care, University of Bristol.

Address for correspondence

William Hamilton, National School for Primary Care Research, Academic Unit of Primary Health Care, University of Bristol, 25 Belgrave Road, Bristol BS8 2AA.

E-mail:w.hamilton@bristol.ac.uk

Submitted: 6 August 2008; Editor's response: 25 August 2008; final acceptance: 6 October 2008.

(OBritish Journal of General Practice.

This article was originally online first on 24 Apr 2009. Cite this article as: Br J Gen Pract 2009; 59: 441-447. Advance online publication. DOI: 10.3399/bjgp09X420860

\section{INTRODUCTION}

Nearly 300000 new cancers are diagnosed in the UK each year, around half of which lead to death. Mortality figures are poor in relation to other developed countries. ${ }^{1,2}$ Considerable investment has been put into improving early diagnosis, in particular the establishment of the '2-week-wait' clinics, and supporting guidance for them from the National Institute for Health and Clinical Excellence (NICE). ${ }^{3}$ These have been accompanied by awareness campaigns and other local initiatives, especially in the more-deprived areas of the UK. Further efforts have been highlighted in the Cancer Reform Strategy, announced in December, 2007. ${ }^{4}$ However, it is not easy to gauge the success of these early diagnosis initiatives, and the value of the 2-weekwait clinics is hotly debated. They do not appear to have improved patient outcomes. ${ }^{5}$

The current emphasis on provision of 2-week-wait clinics has had the unfortunate side-effect of rendering some patients with symptomatic cancer ineligible for rapid investigation. These are the patients with a 'low risk, but not no-risk' symptom. For instance, NICE guidance explicitly states that constipation does not qualify for urgent referral, despite this symptom having a risk of an underlying colorectal cancer of just under $1 \%$ in the over-40s once it has been reported to primary care. ${ }^{6}$ Patients with a low-risk symptom are not rare: less than half of colorectal cancer patients have a symptom qualifying for urgent referral. ${ }^{6}$ Under half of lung cancers take the 2-week-wait route, and less than three-quarters of breast cancers do so. ${ }^{7,8}$ In effect, 2week-wait clinics have created a two-tier service, with a substantial number of patients in the second tier. Not only have these patients missed the rapid diagnostic facility, but waiting times in the routine stream have also risen, so they are worse off. ${ }^{8,9}$ It is even possible that this group comprises those individuals with most to gain from early diagnosis, as staging is worse in patients diagnosed after urgent referral. ${ }^{10}$ Expediting the diagnosis in moreadvanced cancer may not yield a mortality benefit.

The problem of inequalities in access to investigations could be solved by increasing provision of rapid diagnostic services (colonoscopy, ultrasound, perhaps computed tomography (CT) 


\section{How this fits in}

GPs see patients with symptoms that could represent cancer almost daily. From these they select patients for rapid investigation. This process is inexact, and

bedevilled by widely-held misconceptions about the process of cancer

diagnosis. This article highlights five areas of difficulty, and airs possible research and organisational solutions.

scanning) either stationed in primary care, or with direct access from primary care. Alternatively, the guidance for secondary care investigation in the 2week-wait clinics could be liberalised, or even scrapped. For the rest of this article, it is assumed that these are the two main options, and suggestions are made as to what research is needed to help with choosing these. It may be that a single solution may not be possible, as the optimum investigation mode may differ between cancers.

Furthermore, the field of cancer diagnosis has suffered from an unfortunate number of misconceptions, which form the basis of this article. These misconceptions may have contributed to the current imperfect situation in the UK. Some of the misconceptions are widely held, others less so. Although the UK is used as the example, much of the content will pertain to any healthcare system with an emphasis on primary care and the gatekeeper role.

\section{MISCONCEPTION 1: CANCER IS DIAGNOSED IN HOSPITALS}

Specialists do not diagnose cancer - instead they generally confirm (or refute) cancer. Most cancers in the UK present with symptoms, and most initial consultations for these symptoms are in primary care. ${ }^{7,11,12}$ The management of such patients generally follows a schema as illustrated in Figure 1.

Alternative pathways exist, such as when a blood result shows an unexpected iron-deficiency anaemia, triggering consideration of the possibility of colorectal cancer, although such unexpected presentations are relatively rare. Other patients are so ill at their first presentation to medical care that they require emergency admission to hospital, for example with bowel obstruction, chest infection, or urinary retention. ${ }^{7,11,12}$ In these patients, the underlying cancer diagnosis is made while in hospital. Even so, the majority of patients follow the route shown in Figure 1, and most investment in earlier diagnosis has concentrated on this pathway.

Specialist care in 2-week-wait clinics is therefore only involved after cancer has been considered as a possibility and, moreover, the probability of cancer is deemed high enough to warrant investigation. The chance of cancer would also have been discussed with the patient. Thus, specialists in these clinics see patients, not only in whom cancer has been thought possible (or probable), but in whom the site of the cancer has also been considered. In essence, the specialist's diagnostic input is to make the binary decision, 'Does the patient have a cancer at this specific site?'. Definitive diagnosis of cancer requires a biopsy: these are taken in secondary care, apart from the few melanomas inadvertently excised in GPs' surgeries. This requires expertise in performing the definitive test, which may be moderately invasive, like bronchoscopy. However, this is only a small part of diagnosis, with the major parts of the diagnostic pathway having occurred earlier. Of course, as the majority of patients with cancer miss the 2-week-wait clinic, specialists do use diagnostic skills in identifying malignancy. It's odd that these cancers are seen as the 'atypical' ones, and those seen in dedicated cancer diagnostic clinics as the typical ones.

This first misconception has consequences, some of them serious. The most important is that data derived from secondary care are used to guide decisions in primary care. The NICE guidance was based on a thorough literature review - mostly of secondary care research, as there was very little primary care data available at the time. There is much more now, with fairly convincing figures of the risk of cancer with symptoms of colorectal, prostate, lung, and brain tumours, plus symptoms such as dysphagia and haematuria. ${ }^{6,13-17}$ However, when secondary care data are applied to the primary care population, surprising things happen. Patients with

\begin{tabular}{|c|c|c|c|}
\hline $\begin{array}{l}\text { The patient has } \\
\text { some feature that } \\
\text { makes cancer a } \\
\text { possibility }\end{array}$ & $\begin{array}{l}\text { The GP makes } \\
\text { a judgement } \\
\text { of the } \\
\text { likelihood } \\
\text { of cancer }\end{array}$ & $\begin{array}{l}\text { A primary care } \\
\text { test suggests the } \\
\text { risk of cancer } \\
\text { warrants referral }\end{array}$ & $\begin{array}{l}\text { Following } \\
\text { referral, a biopsy } \\
\text { is undertaken, } \\
\text { confirming (or } \\
\text { refuting) cancer }\end{array}$ \\
\hline
\end{tabular}


diarrhoea in colorectal clinics have a positive predictive value for colorectal cancer of $7.7 \% .{ }^{18}$ In general practice it is $1.1 \%,{ }^{6}$ and in the community it will be much lower again. ${ }^{19}$ This is demonstrated by a questionnaire study of unselected, apparently healthy, 50-80 year olds in two practices: $12 \%$ qualified for an urgent referral for possible colorectal cancer. ${ }^{20}$ Decision aids derived from secondary care data, like the Selva score for colorectal cancer, ${ }^{18}$ will have poor performance characteristics if used in primary care.

As selection of patients for investigation is initiated in primary care, it follows that research on this topic must be based in primary care. Knowing the predictive values of individual symptoms or, better still, combinations of symptoms, would allow better criteria for selection of patients. These criteria could guide either of the two main options for improved diagnosis - open-access GP investigations or liberalised access to specialist opinion. However, given that both options would require an increase in total investigations (as lowrisk patients are now being investigated), it may be simpler to offer a wider range of direct-access investigations. Open-access investigations could be guided by rules based on primary care data. Indeed, as many endoscopies are now performed by nonmedical staff, allowing greater access to direct investigations may free up specialist time to see a smaller number of patients with more-complex clinical features.

\section{MISCONCEPTION 2: GPS ARE NOT VERY GOOD AT DIAGNOSING CANCER}

GPs' failure (or perceived failure) to diagnose cancer is a major cause of litigation, accounting for $14 \%$ of negligence claims against them. ${ }^{21}$ The problem for GPs is that for every 'missed cancer' there are several patients with similar symptoms who do not have cancer, and who have been spared the worry of investigation. Litigants, and their lawyers, concentrate on the false negatives and ignore the true negatives. Newspapers also condemn GPs, misquoting the UK cancer director to beef up their story:

'Patients are dying of cancer because GPs are failing to identify their symptoms, the government's top cancer expert has warned. Professor Mike Richards said botched diagnoses were now "a significant concern". 222

What evidence is there that GPs are poor at cancer diagnosis? For most patients the primary care element of diagnosis is quick: the median interval between first symptom of colorectal cancer presented to the GP and referral for investigation is 6 days, and the mean number of consultations before referral is one. ${ }^{12}$ This fits very well with an initial consultation, at which blood tests are ordered, and the patient being referred at the review consultation - all within 1 week. Significant delays largely only follow presentations with anaemia and abdominal pain, and these presentations have a worse mortality. ${ }^{23}$ The figures are not quite as good for lung cancer, with a median of 51 days from first symptom presentation to diagnosis. ${ }^{7}$ However, most GPs will have taken a chest X-ray (CXR) before referral. This X-ray is usually deferred until the effect of initial treatment is assessed (unless the patient has haemoptysis), so this 51-day figure may be acceptable. However, as with colorectal cancer, one concern is the patients whose symptoms do not prompt the GP to request a CXR, and who incur delays as a result. Only $66 \%$ of a recent series of lung cancers had had a primary care CXR before diagnosis. ${ }^{24}$ One useful strand of research will be examining those patients whose diagnosis is delayed - do they have particular symptom patterns that mislead the clinician? Additionally, it is possible that lowering the threshold for a CXR could expedite the diagnosis for a small number of patients with lung cancer, although research will need to address whether the benefits outweigh the costs (both economic and clinical) of additional X-rays.

One thing most doctors have - which is almost impossible to research - is an innate sense that their patient is ill. The precise diagnosis may not be apparent, but the fact there is one is apparent. This has been shown in other diagnostic fields, such as childhood fever. ${ }^{25}$ The most powerful predictor of cancer is not a particular symptom or test, but a GP's summation of all the subtle clues present in the consultation. A clue of how strong this summation is comes from studies of the GP records of patients with lung, colorectal, or prostate cancer. When a variable for a GP's note of possible cancer (generally written as '?cancer') was added to a multivariable equation containing all the other significant symptoms of cancer, the odds ratio for this new variable much outweighed all the other variables. ${ }^{26}$ This may represent GPs identifying clinical features not conventionally considered relevant - like the patient's tone of voice, or a subtle change in their appearance. Clinicians understand such subtleties, which make up the art of medicine, rather than its science.

One proposed solution to the perception that GPs are missing cancers is further postgraduate education. However, education in this field is still dominated by secondary care. Yet secondary care 
doctors only see selected patients, so are relatively inexperienced with the selection process. A recent $B M J$ review of colorectal cancer followed this pattern, in that the brief diagnosis section only referenced one 1993 secondary care paper, and two consensus documents. ${ }^{27} \mathrm{~A}$ second example of misdirected education relates to lung cancer. Cough is the commonest symptom, reported by two-thirds of patients to their GPs before diagnosis. ${ }^{15}$ Yet all textbooks - and the NICE guidelines - put haemoptysis at the top of their list. Certainly, haemoptysis has the highest predictive value of the common symptoms of lung cancer, but it is only reported by $20 \%$ of patients to primary care before diagnosis..$^{15}$ Concentrating on it would mean that $80 \%$ of lung cancers would be missed. Similar considerations pertain to rectal bleeding and colorectal cancer, although the figure here is around $40 \%{ }^{6}$ This is no argument against continuing education, but it has to be based on primary care research. Therefore, it is encouraging that the forthcoming national audit of cancer diagnosis plans to incorporate significant event audits of cancer diagnoses at a practice level.

\section{MISCONCEPTION 3: SCREENING MAKES SYMPTOMATIC DIAGNOSIS LESS RELEVANT}

There is an under-appreciation of the importance of symptomatic presentation of cancer, although why this should be so is less clear. In part, I suspect it is wishful thinking that screening will obviate the problem. In the UK, there is a screening programme for only three cancers: two common ones, breast and colorectal, plus one rarer cancer, cervical. These programmes have reduced mortality - or will do. ${ }^{28,29}$

Prostate screening is performed in many countries, but without convincing evidence of a mortality benefit, and it is not recommended in the UK..$^{30}$ However, each screening programme has its target age groups, with cancers occurring outside these ages; they also have partial uptake and less than $100 \%$ sensitivity. Colorectal screening is a good example, with only $40 \%$ of cancers occurring within the age range chosen for screening, and most of the missing cases being older. ${ }^{19}$ Uptake of screening was reported to be $60 \%$ in the first round, and $52 \%$ in the second round. ${ }^{31}$ The figures for other countries, such as the US, are worse. ${ }^{32}$ The sensitivity of colorectal screening using faecal occult blood tests is around $60 \% .{ }^{30}$ Multiplying the age factor $40 \%(0.4)$ by the take-up factor of $60 \%$ (0.6) by the sensitivity of $60 \%(0.6)$, gives $14 \%$ of colorectal cancers detectable by screening. This figure may be pessimistic, as some polyps are detected by screening: removal of these may reduce cancer mortality later. Therefore, perhaps at best, $20 \%$ of colorectal cancers will be detected (or prevented) by screening. Recent figures from the Midlands of England bear these calculations out: in the fifth year of screening, 31 of a total of 200 cancers were identified through the screening programme. ${ }^{33}$ Similar figures pertain to breast cancer: $75 \%$ of breast cancers are diagnosed outside the mammography programme, for similar reasons. Cervical cancer is an exception, as sensitivity is higher, the age range for screening is wider, and uptake is $72 \% .{ }^{27}$ Overall, of the 300000 new cancers each year in the UK, perhaps 7000 colorectal, 14000 breast and 2000 cervical tumours will be identified by screening, leaving over $90 \%$ of the total to present symptomatically.

Screening is useful, with well-accepted mortality benefits - but it is not cost-free. The estimated cost for the first year of colorectal cancer screening alone is $£ 112.8$ million. ${ }^{34}$ This is a major opportunity cost, in part borne by symptomatic diagnosis, as there are only a limited number of colonoscopists. Similarly, research resources for symptomatic cancer compete with those for screening. The perception that symptomatic diagnosis is decreasing in relevance makes it harder to fulfil the research agenda outlined in the first two misconceptions.

\section{MISCONCEPTION 4: THE CANCER DETECTION RATE IN CLINICS SHOULD BE HIGH}

No target detection rate has been published, but there is clear unhappiness that the proportion of 2week-wait referrals who actually have cancer has fallen from around $13 \%$ to $5 \% .{ }^{8}$ Similar figures pertain to colorectal cancer. ${ }^{35}$ GPs are accused, probably correctly, of gaming the system by ensuring their patient qualifies for a 2-week-wait referral by fudging the NICE criteria. ${ }^{35,36}$ At the same time, there is widespread concern that too many cancers miss the 2-week-wait route, and that they are diagnosed more slowly by following the other routes. $^{8}$

However, to increase the number diagnosed within the 2-week-wait clinics requires a fall in the percentage of 2-week-wait referrals that are positive. This is the usual trade-off between sensitivity and specificity. This is particularly relevant to colorectal cancer, which is probably the most difficult cancer for selection of patients to investigate. This difficulty stems from the fact that there is no primary care test to identify which patients presenting with a low-risk symptom are at a higher risk of cancer - the third box in Figure 1. There is such a test for lung (the CXR), for prostate 
(the prostate-specific antigen [PSA]), and for breast (the presence of a lump) cancers. ${ }^{37}$ Thus, clinics for these three cancers may well be able to maintain a high positivity rate, without serious loss of sensitivity.

Not so colorectal cancer. The majority of patients only have a low-risk symptom. ${ }^{38}$ Aiming for high specificity in 2-week-wait referrals means that all low-risk (say below $5 \%$ ) symptoms would not qualify for urgent investigation. This means no referrals other than masses, severe anaemia, and debatably - rectal bleeding qualify. ${ }^{6}$ Restricting referrals to those groups would dramatically improve the efficiency of the clinic. But what about the man aged 70 years with a haemoglobin of $10.8 \mathrm{~g} / \mathrm{dl}$, and a risk of cancer of $3.2 \% ?^{39}$ Is he not worthy of rapid investigation?

Research efforts should focus not just on calculation of the initial risk of cancer with symptoms, but on how this risk can be refined, ideally using primary care tests. This is especially relevant to colorectal cancer. Some progress is being made in development of scoring systems, ${ }^{38}$ use of serum tumour markers, ${ }^{40}$ and measurement of free rectal $D N A,{ }^{41}$ but none of these initiatives has yet been trialled in the low-incidence population of primary care. Until one or more of these methods is found to be successful, an increase in sensitivity at the 2-week-wait clinic must be accompanied by a parallel fall in specificity. Colorectal 2-week-wait clinics will have to accept a lower strike rate, including the resource implications of all these extra investigations. Fortunately, there is economic support for such a move. A recent UK study modelled 13 possible improvements across the whole diagnostic and treatment spectrum for colorectal cancer. A lowering in referral threshold gave the second-highest possible benefit. ${ }^{33}$

\section{MISCONCEPTION 5: THERE IS NO PROOF THAT EARLY DIAGNOSIS OF SYMPTOMATIC CANCER MATTERS}

Almost everyone believes that early diagnosis of symptomatic cancer saves lives. The 2-week-wait clinics and other diagnostic initiatives were established on this assumption (although psychological benefits from reducing delays are also relevant). At the same time, some clinicians and researchers are nihilistic about whether genuine benefits are available. This nihilism is particularly common in lung cancer.

The argument is that there are no randomised controlled trials examining benefits from symptomatic diagnosis of cancer - perhaps because expediting diagnosis is deemed so obviously desirable. This leaves observational data.
However, observational studies cannot test the hypothesis that expediting diagnosis provides a mortality benefit. Observational studies have additional problems, such as the definition of a symptom, and of its timing, let alone the issues of confounding and biases.

Some aspects are uncontroversial, however. The relationship between staging and prognosis is well accepted. It is also clear that cancers progress through the various stages, although probably not linearly. The problem is fitting symptoms onto that timetable. For some cancers, it may be that the window of opportunity for curative treatment may occur before symptoms appear, making early symptomatic diagnosis of little, or no, mortality benefit. This may be largely true for lung cancer. However, a systematic review of observational studies in breast cancer found reduced survival with diagnostic delays of 3-6 months. ${ }^{42}$ This is not quite the same as proving that reducing diagnostic delays helps, but it is very consistent with that view.

Further evidence is available from studies of treatment delay: local recurrence of cancer is more common with longer waiting times for radiotherapy. ${ }^{43,44}$ Even in the interval between diagnostic and treatment planning scans (median 28 days), two-thirds of patients with head and neck cancer have a demonstrable increase in tumour size, with $20 \%$ developing new lymph node metastases..$^{45}$ If cancer progresses this rapidly after diagnosis, it is not unreasonable to assume it progresses in the time spent establishing a diagnosis.

It is also possible to study the effects of early diagnosis in trials, even though that has not been done. If, for example, any of the early diagnostic initiatives for colon cancer outlined in misconception 4 continue to show promise, they can be compared to standard care. These trials should include as an endpoint not just time to diagnosis but mortality, plus 'softer' outcomes such as reductions in anxiety. Such trials will be expensive because they will be large. Even so, it is conceivable this final misconception can be debunked - for some cancers at least. Moreover, even if it is not possible to prove, to the satisfaction of everyone, that early diagnosis matters, it is hard to mount an argument for delayed diagnosis.

To return to the beginning: the UK has seen progress in prevention, screening, and treatment of cancer. There are more diagnostic facilities on offer, but this has brought its own problems. More research is needed as well as clinical efforts towards identifying those truly at risk of cancer, and providing facilities for them. As a spin-off, enough evidence may be generated to show that early symptomatic diagnosis is of genuine value. 


\section{COMMENTARY}

\section{The truth behind 'five misconceptions'}

I agree with William Hamilton that strategies to detect cancer should aim for evidence-based efficiency, and that more of the evidence should come from community-based research. His commentary is a thoughtful discussion of ways of improving cancer diagnosis in the context of current strategies of the British NHS. ${ }^{1}$ But the scaffolding of the argument puzzled me: I recognise none of the five misconceptions that he argues are impeding progress in improving cancer diagnostic services. And so, in the spirit of friendly debate, I offer my contrarian perceptions of Hamilton's misconceptions.

Misconception 1: 'cancer is diagnosed in hospitals'. This doesn't mean much to me as a concept - the familiar pathway to cancer diagnosis that Hamilton describes looks like a reasonably rational team-based approach to achieving the answers the patient needs. When a patient comes to me with symptoms that might represent cancer, I use the resources at my disposal as a GP, the specialist those at theirs, and together we, hopefully, rule cancer in or out, preferably according to a shared guideline. Hamilton argues that the perception that secondary care is responsible for diagnosis leads to GPs using the wrong data when making referral decisions. This may well be the case, but, as he points out, the positive predictive values of most symptoms of cancer are lower in primary care than in referred populations. Guidelines based on predictive values derived in community populations will, if anything, increase the threshold for referral. Primary care research may show just how difficult it is for clinicians to decide which patients with diarrhoea to refer, but is unlikely to bolster the argument for expanding access to investigation for the low but not no-risk patients that Hamilton describes later in his discussion.

Misconception 2: GPs 'are thought' to be poor at cancer diagnosis. Who, other than media journalists, thinks this? It is certainly not our hospital colleagues. I regularly run joint medical student teaching sessions with consultant colleagues where we work through cases from first primary care presentation to final diagnosis and treatment. To a man and woman, these specialists acknowledge the difficulties facing patients and GPs in making rational decisions about referral in low risk populations. There is always room for better education, but I can't accept the argument that taking seriously the symptom of haemoptysis means that I will then miss lung cancer by ignoring patients with cough. It is possible for clinicians to hold more than one thing in their mind at one time, and as Hamilton has himself shown, the integrative judgement of the GP is often the most powerful factor in making correct decisions about cancer risk.

Misconception 3: There is 'an under-appreciation of the importance of symptomatic diagnosis'. Where is the evidence? Screening has a role in cancer diagnosis, but GPs' involvement with cancer diagnosis continues to be mainly in patients with symptoms that worry them. I don't accept the argument that the existence of mammography and cervical screening programmes means I will act with any less diligence in evaluating a woman who comes to me with a breast lump or inter-menstrual bleeding.

Misconception 4: 'the cancer detection rates in clinics ought to be high'. For the reasons Hamilton gives, I agree that this statement is incorrect, but I don't know anyone who holds this belief. Surgeons, for example, were influential in liberalising the NICE guidance for investigation of dysphagia, so as not to exclude from investigation patients with curable oesophageal cancer who lacked red flag symptoms.

Misconception 5: 'there is no proof that early diagnosis of symptomatic cancer matters'. As Hamilton points out, this is not a widely held perception - almost everyone believes that early diagnosis is a good thing and behaves accordingly. Whether or not treatment improves prognosis, patients and doctors want speedy diagnosis of concerning symptoms to resolve uncertainty and plan treatment. It would be satisfying to have better evidence that we can reduce mortality by swift action, but I do not accept that patients or doctors slow down because they lack such proof.

\section{Tim Lancaster,}

Director of Clinical Studies, The Medical School Office, The John Radcliffe, Headington, Oxford, OX3 9DU. E-mail: Tim.lancaster@dphpc.ox.ac.uk

\section{Provenance}

Commissioned; not peer reviewed.

\section{REFERENCE}

1. Hamilton W. Five misconceptions in cancer diagnosis. Br J Gen Pract 2009; 59: 441-447.

\section{Funding body}

William Hamilton is funded through his research practice (Barnfield Hill, Exeter) and an NCCRCD postdoctora fellowship. He also receives funding from the Department of Health's NIHR School for Primary Care Research funding scheme. The views expressed in the publication are those of the author and not necessarily those of the Department of Health 


\section{Ethics committee}

Ethical approval was not required

\section{Competing interests}

William Hamilton is a primary care cancer researcher, so would welcome any increase in funding to this area. He has missed at least one cancer diagnosis

\section{Acknowledgements}

This paper has benefited from the input of several colleagues, including Richard Neal, David Kessler, Christine Campbell, and two British Journal of General Practice referees who pointed me to the evidence relating to treatment delays. I thank them all.

\section{Discuss this article}

Contribute and read comments about this article on the Discussion Forum: http://www.rcgp.org.uk/bjgp-discuss

\section{REFERENCES}

1. Gatta G, Capocaccia R, Sant M, et al. Understanding variations in survival for colorectal cancer in Europe: a EUROCARE high resolution study. Gut 2000; 47(4): 533-538.

2. Gatta G, Ciccolallo L, Capocaccia R, et al. Differences in colorectal cancer survival between European and US populations: the importance of sub-site and morphology. Eur J Cancer 2003; 39(15): 2214-2222.

3. National Institute for Health and Clinical Excellence. Referral guidelines for suspected cancer. London: National Institute for Health and Clinical Excellence, 2005.

4. Department of Health. Cancer reform strategy. London: Department of Health, 2007.

5. Jiwa M, Saunders C. Fast track referral for cancer. BMJ 2007; 335(7614): 267-268.

6. Hamilton W, Round A, Sharp D, Peters T. Clinical features of colorectal cancer before diagnosis: a population-based case-control study. Br I Cancer 2005; 93(4): 399-405.

7. Barrett J, Hamilton W. Pathways to the diagnosis of lung cancer in the UK: a cohort study. BMC Fam Pract 2008; 9: 31

8. Potter S, Govindarajulu S, Shere M, et al. Referral patterns, cancer diagnoses, and waiting times after introduction of two week wait rule for breast cancer: prospective cohort study. BMJ 2007; 335(7616): 288-291.

9. Sauven P. Impact of the ' 2 week wait' on referrals to breast units in the UK. Breast 2002; 11(3): 262-264.

10. Neal RD, Allgar VL, Ali N, et al. Stage, survival and delays in lung, colorectal, prostate and ovarian cancer: comparison between diagnostic routes. Br J Gen Pract 2007; 57(536): 212-219.

11. Barrett J, Hamilton W. Pathways to the diagnosis of prostate cance in an English city: a population based survey. Scand J Urol Nephrol 2005; 39(4): 267-270.

12. Barrett J, Jiwa M, Rose P, Hamilton W. Pathways to the diagnosis of colorectal cancer: an observational study in three UK cities. Fam Pract 2006; 23(1): 15-19.

13. Hamilton W, Kernick D. Clinical features of primary brain tumours: a case-control study using electronic primary care records. $\mathrm{Br} J \mathrm{Gen}$ Pract 2007; 57(542): 695-699.

14. Hamilton W, Sharp D, Peters TJ, Round A. Clinical features of prostate cancer before diagnosis: a population-based case-control study. Br J Gen Pract 2006; 56(531): 756-782.

15. Hamilton W, Peters TJ, Round A, Sharp D. What are the clinical features of lung cancer before the diagnosis is made? A population based case-control study. Thorax 2005; 60(12): 1059-1065.

16. Jones R, Latinovic R, Charlton J, Gulliford MC. Alarm symptoms in early diagnosis of cancer in primary care: cohort study using General Practice Research Database. BMJ 2007; 334(7602): 1013-1014.

17. Du Toit J, Hamilton W, Barraclough K. Risk in primary care of colorectal cancer from new onset rectal bleeding: 10 year prospective study. BMJ 2006; 333(7558): 69-70.

18. Selvachandran S, Hodder R, Ballal M, et al. Prediction of colorecta cancer by a patient consultation questionnaire and scoring system: a prospective study. Lancet 2002; 360(329): 278-283.

19. Hamilton W, Sharp D. Diagnosis of colorectal cancer in primary care the evidence base for guidelines. Fam Pract 2004; 21(1): 99-106.
20. Soin G, Armitage J, McKay J, et al. Colorectal symptoms in the community - a ticking time bomb? Gut 2004; 53(suppl 3): abstract 069.

21. Lalanda M. Missed opportunities. Medical Protection Society: Casebook 2007; 14(3): 8-11.

22. Campbell D. GPs warned over failure to diagnose cancers. The Observer, 2008. http://www.guardian.co.uk/society/2008/apr/ 13/nhs.cancerl (accessed 25 Feb 2009).

23. Stapley S, Peters TJ, Sharp D, Hamilton W. The mortality of colorectal cancer in relation to the initial symptom and to the duration of symptoms: a cohort study in primary care. Br J Cancer 2006; 95(10): 1321-1325.

24. Stapley S, Sharp D, Hamilton W. Negative chest X-rays in primary care patients with lung cancer. Br J Gen Pract 2006; 56(529): 570-573.

25. Monteny M, Berger M, van der Wouden J, et al. Triage of febrile children as a GP cooperative: determinants of a consultation. $\mathrm{Br}$ Gen Pract 2008; 58(549): 242-247.

26. Hamilton W. Towards earlier diagnosis of cancer in primary care: population-based case-control study of colorectal, lung and prostate cancer. MD thesis, University of Bristol, 2005.

27. Ballinger A, Anggiansah C. Colorectal cancer. BMJ 2007; 335(7622): 715-718.

28. Peto J, Gilham C, Fletcher O, Matthews FE. The cervical cancer epidemic that screening has prevented in the UK. Lancet 2004; 364(9430): 249-256.

29. Towler B, Irwig L, Glasziou P, et al. A systematic review of the effects of screening for colorectal cancer using the faecal occult blood test, hemoccult. BMJ 1998; 317(7158): 559-565.

30. Frankel S, Davey Smith G, Donovan JL, Neal DE. Screening fo prostate cancer. Lancet 2003; 361(9363): 1122-1128.

31. Weller D, Coleman D, Robertson R, et al. The UK colorectal cance screening pilot: results of the second round of screening in England. Br J Cancer 2007; 97(12): 1601-1605.

32. Seeff L, Nadel M, Klabunde C, et al. Patterns and predictors of colorectal cancer test use in the adult U.S. population. Cancer 2004; 100(10): 2093-2103.

33. Goodyear SJ, Leung E, Menon A, et al. The effects of populationbased faecal occult blood test screening upon emergency colorectal cancer admissions in Coventry and north Warwickshire. Gut 2008; 57(2): 218-222.

34. York Health Economics Consortium. Bowel cancer services: costs and benefits. London: Department of Health, 2007.

35. Rai S, Kelly MJ. Prioritization of colorectal referrals: a review of the 2-week wait referral system. Colorectal Dis 2007; 9(3): 195-202.

36. Freeman VG, Rathore SS, Weinfurt KP, et al. Lying for patients: physician deception of third-party payers. Arch Intern Med 1999; 159(19): 2263-2270.

37. Barton MB, Elmore JG, Fletcher SW. Breast symptoms among women enrolled in a health maintenance organization: frequency, evaluation, and outcome. Ann Intern Med 1999; 130(8): 651-657.

38. Hamilton W. Derivation of a score for identifying colorectal cancer in primary care. Gut 2007; 56(suppl 2): A49.

39. Hamilton W, Lancashire R, Sharp D, et al. The importance of anaemia in diagnosing colorectal cancer: a case-control study using electronic primary care records. Br J Cancer 2008; 98(2): 323-327.

40. Hurst NG, Stocken DD, Wilson S, et al. Elevated serum matrix metalloproteinase 9 (MMP-9) concentration predicts the presence of colorectal neoplasia in symptomatic patients. Br J Cancer 2007; 97(7): 971-977.

41. Loktionov A. Cell exfoliation in the human colon: myth, reality and implications for colorectal cancer screening. Int J Cancer 2007; 120(11): 2281-2289.

42. Richards MA, Sainsbury J, Ramirez A, et al. Delay in diagnosis in breast cancer. Lancet 1999; 353(9159): 2155-2162.

43. Chen Z, King W, Pearcey R, et al. The relationship between waiting time for radiotherapy and clinical outcomes: A systematic review of the literature. Radiother Oncol 2008; 87(1): 3-16.

44. MacKillop WJ. Killing time: the consequences of delays in radiotherapy. Radiother Oncol 2007; 84(1): 1-4.

45. Jensen AR, Nellemann HM, Overgaard J. Tumor progression in waiting time for radiotherapy in head and neck cancer. Radiother Oncol 2007; 84(1): 5-10. 